
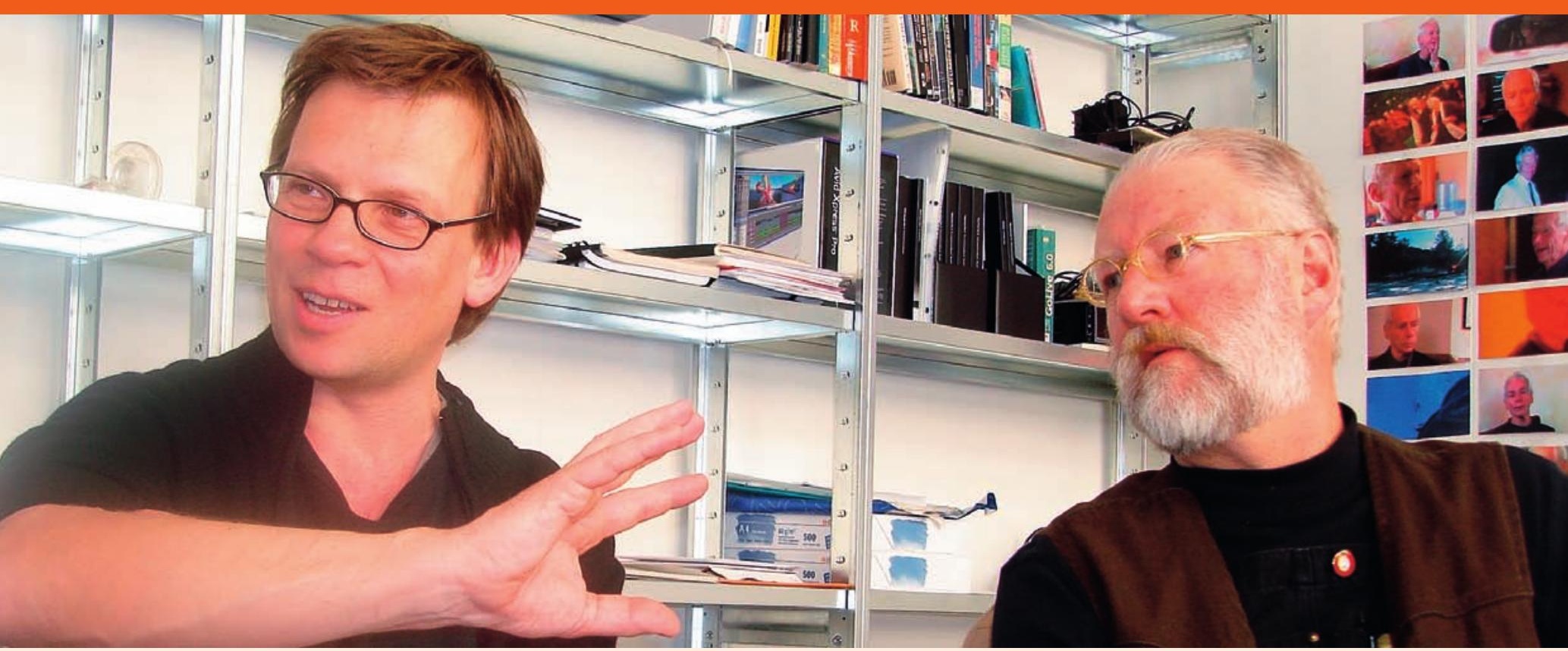

Im Gespräch: Edgar Hagen (links), Autor des Films «Someone beside you», und Eric Chapin, langjähriger Psychotherapeut im Windhorse-Projekt.

Foto: BK

Gespräch zum Film «Someone beside you»

\title{
«Vom Traum zurück in die Wirklichkeit»
}

\author{
«Someone beside you» ist das Resultat einer langjährigen Auseinandersetzung \\ des Filmemachers und Philosophen Edgar Hagen mit dem Thema Psychose. \\ Im folgenden Gespräch äussern sich der Autor sowie der Psychotherapeut \\ Eric Chapin zum Film und seiner Entstehungsgeschichte und beleuchten aus \\ ihrer Sicht wesentliche Aspekte des Phänomens «Psychose». Chapin arbeitet \\ seit über 30 Jahren mit psychotischen Patienten, davon 13 Jahre im Wind- \\ horse-Projekt in Boulder, wo ein Teil des Films entstanden ist.
}

Gesprächsmoderation: Bruno Kesseli
SÄZ: Herr Hagen, Sie sind Filmemacher und Philosoph. Nun ist das Phänomen Psychose, mit dem Sie sich schon länger auseinandersetzen, nicht eben ein klassischer Gegenstand der Philosophie. Wie erklärt sich Ihr Interesse an diesem Thema?

Edgar Hagen: Die Philosophie, so wie sie in unseren Breiten praktiziert wird, bewegt sich in der Regel mehr oder weniger im luftleeren Raum, sie ist ohne direkte Anwendung. Ich finde es dagegen spannend, philosophische Fragestellungen in eine «Lebenswirklichkeit» herunterzuholen. Vor dem Hintergrund der Existenzphilosophie kann man die Psychose ohne weiteres als eine Extremform der menschlichen Existenz begreifen. Extrembereiche der menschlichen Existenz, insbesondere auch radikale ethische Fragestellungen, interessieren mich, seit ich denken kann. Insofern ist meine filmische Auseinander- setzung mit dem Thema Psychose nur die Fortsetzung einer langen Auseinandersetzung mit extremen Geisteszuständen. Letztlich steht dahinter der Versuch, die menschliche Existenz in ihrer Gesamtheit zu begreifen.

Nun gibt es verschiedenste Arten von extremen Geisteszuständen. Gab es in Ihrem Fall eine Art Schlüsselerlebnis, das speziell psychotisches Erleben in den Fokus rückte?

Hagen: Zum einen habe ich zunehmend gespürt, dass mein Weg da entlanggeht, wo die Angst sitzt. Und zwar insbesondere die Angst von Menschen, die ausgegrenzt sind. Eine Art Schlüsselerlebnis gibt es vielleicht tatsächlich: Während meiner Studienzeit in Berlin, wo ich bis 1987 studiert habe, kam ich täglich an einer Suppenküche vorbei, vor der mittags rund 200 Randstän- 
dige warteten. Die Szenerie hat mich sehr berührt, und eines Tages stellte ich mich in der Schlange an und trat über die Schwelle. Dort hineinzugehen, die Angst vor dem unbekannten Phänomen zu überwinden, war für mich entscheidend. Die Welt, die sich mir eröffnete, war ungemein lebendig, farbig, anders, als ich sie erwartet hatte. Es entstand der Wunsch, solche unbekannten Welten auch anderen zu vermitteln.

1998 realisierten Sie dann mit dem kurzen Dokumentarfilm «Dorothea Buck - vom Wahn zum Sinn» ihre erste Arbeit als Filmemacher zum Thema Psychose.

Hagen: Als ich Dorothea Buck 1994 kennenlernte, war dies ein weiteres Schlüsselerlebnis für mich. Sie konnte mir auf einzigartige Weise vermitteln, welche Dimensionen sich in einer Psychose auftun. Damit verschaffte sie mir Zugang zu einer Welt, für die wir normalerweise kein Verständnis haben, obwohl sie in jedem von uns drinsteckt.

Versuchen wir, diese Welt etwas genauer zu bestimmen. Herr Chapin, Sie arbeiten seit über 30 Jahren intensiv mit psychotischen Patienten. Gibt es aus Ihrer Sicht so etwas wie eine Essenz der Psychose?

Eric Chapin: Keine einfache Frage ... psychotisches Erleben hat so viele Dimensionen, bei denen man ansetzen könnte. Die wissenschaftliche Auseinandersetzung mit dem Phänomen «Psychose» ist in der Regel auf die Biologie des Gehirns fokussiert. Die auf diese Weise gewonnenen Erkenntnisse sind sehr wichtig, und wir schliessen sie in unsere Arbeit ein. Im Windhorse-Projekt haben wir aber den Fokus zunehmend auf das Zusammenspiel von Subjektivität und Biologie gerichtet. Wir sind zum Schluss gekommen, dass die subjektive Erfahrung des Wahnsinns, des Verrückt-Seins, für sich genommen ein wesentlicher Faktor ist, der die biologischen Funktionen des Gehirns stört. Die subjektive Erfahrung der Psychose führt zu einem biologischen Ungleichgewicht. Das psychotische Individuum sucht nach Strategien, um seinen Zustand zu verbessern. In der Regel verstärken diese unbewusst angewandten Techniken jedoch die Störung, statt sie zu beseitigen. Anstelle von Gesundheit schaffen sie Krankheit. Diese Dysbalance folgt einer Logik, die man verstehen und erforschen kann.

Das tönt einleuchtend, aber ziemlich abstrakt. Können Sie diese Überlegungen anhand von Beispielen konkretisieren?

Chapin: Vielleicht ist es hilfreich, die Sache umgekehrt zu betrachten. Wenn wir mit psychotischen Menschen arbeiten, versuchen wir mit verschiedenen Techniken, ihren beschleunigten Geist zur Ruhe zu bringen. Je nach Patient kann dies zum Beispiel mittels Meditation, Tai-Chi, Jogging oder dem Aufräumen des Zimmers geschehen. Die Patienten suchen selber auch nach solchen Strategien, aber sie verschlimmern damit ihren Zustand: statt sich zu beruhigen, geraten sie in Panik oder werden aggressiv. Hier muss die therapeutische Unterstützung ansetzen.

Im Film werden in verschiedenen Statements - auch von Ihnen - Parallelen zwischen psychotischem Erleben und Traum betont.

Chapin: In der Tat kann das Traumerleben als eine Art psychotisches Erleben gesehen werden. Im Traum tun wir verrückte Dinge, wir fliegen umher oder würgen andere Leute. Normaler-

\section{«ln der Tat kann Traumerleben als eine Art psychotisches Erleben gesehen werden»}

weise schütteln wir diese Welt nach dem Erwachen mit der ersten Tasse Kaffee ab. Wir haben im Alltag viele Strategien entwickelt, um auf dem Boden zu bleiben. Der Psychotiker schafft diesen Übergang nicht, er nimmt die Realität wie im Traum wahr und verhält sich entsprechend. Wir versuchen in unserer Arbeit, ihm auf dem Weg vom Traum zurück in die Wirklichkeit behilflich zu sein.

Hagen: Bei Filmen zum Thema Psychose ist mir aufgefallen, dass meist der Weg in die Psychose nachgezeichnet wird. Dies hängt wahrscheinlich mit unserer Kultur zusammen, die nach starken, dramatischen Geschichten verlangt. Mich dagegen hat die umgekehrte Richtung in ihren Bann gezogen: Wenn der Weg aus der Psychose möglich ist, so birgt das Nachzeichnen dieses Wegs eine mindestens so starke Spannung und Faszination wie der Weg in die Psychose hinein. Am Schluss dieses Wegs steht ja nicht eine heile Welt, sondern eine neue Form, in der Welt zu stehen.

Im Konzept von Windhorse spielt das Milieu, in dem sich die Patienten aufhalten, für den Weg aus der Psychose eine zentrale Rolle.

Chapin: Psychotische Menschen reagieren extrem sensibel auf ihre Umgebung. Wenn nun die Umgebung ungesund ist, wie sollen da die Patienten gesunden? Wie sollen Patienten innerlich zur Ruhe kommen, wenn ihnen von allen Seiten dauernd eingetrichtert wird, sie würden 
nie mehr gesund, sie müssten sich mit einer beschränkten Existenz zufriedengeben. Natürlich hat eine solche Sichtweise ihre Berechtigung aber eine solche Botschaft vermittelt keine Hoffnung, keinen Mut. Was die Patienten brauchen, sind positive Signale: «Du kannst an deinem Geist arbeiten, du kannst gesund werden: mit Hilfe von Medikamenten, mit Unterstützung und Verständnis durch die Menschen in Deiner Nähe und durch eine dir zuträgliche Umgebung.» Das ist übrigens kein neuer Ansatz, sondern uraltes Wissen.

Hagen: Bei der Realisierung des Films ist mir aufgefallen, dass wir in unserer Gesellschaft nicht lernen, mit Ausnahmezuständen des Geistes umzugehen. Die Beschleunigungen des Geistes, von denen im Film die Rede ist, erleben ja schon Kinder. Eigentlich sollte es zur Grundausbildung in der Schule gehören, zu lernen, den Geist zu verlangsamen, zur Ruhe zu bringen. Wenn Kinder in der Schule Schwierigkeiten machen, heisst es in der Regel aber nur «stopp!». Ich sehe das als ein kulturelles Problem.

Chapin: Nimmt man eher den medizinischen, den psychiatrischen Bereich, sehe ich ebenfalls eine Art kulturelles Problem darin, dass wir in der Ausbildung fast ausschliesslich darauf trainiert werden, das Pathologische zu sehen, das, was nicht funktioniert. Das ist natürlich da, aber daneben ist eben nach wie vor sehr viel Schönes, sehr viel Gesundes vorhanden. Der psychotische Patient hat immer noch seine künstlerischen Fähigkeiten, seine Sensibilität, sein feines Gehör, seine Nase. Manchmal hat er sogar «SuperOhren» oder eine «Super-Nase». Wir trainieren unsere Therapeuten darin, diese Eigenschaften

\section{«Trust your mind: das ist eine zentrale Botschaft, die wir unseren Patienten zu vermitteln versuchen»}

zu sehen und gleichzeitig die Extreme in sich selbst wahrzunehmen. Patient und Therapeut begegnen sich dadurch auf einem annähernd gleichen Niveau. Gelegentlich werden wir wegen dieses Ansatzes in die Anti-Psychiatrie-Ecke gestellt, allerdings vorwiegend von Leuten, die unsere Arbeit nicht wirklich kennen. In der Realität ist es umgekehrt: Wir sind pro Psychiatrie. Hagen: Was Eric eben gesagt hat, ist auch im Film ein wichtiger Punkt. Es geht darum, zu zeigen, dass beim Gesunden und beim Kranken das Gemeinsame überwiegt. Derjenige, der die Diagnose stellt, ist gar nicht so weit von dem entfernt, der sie erhält.

Diese Haltung führt offenbar dazu, dass man dem Gegenüber sehr viel zutraut. Im Film gibt es diesbezüglich eindrückliche Szenen, etwa die Rückkehr von Karen an den Ort ihres Suizidversuchs oder die Auseinandersetzung zwischen Kaspar und Jakob

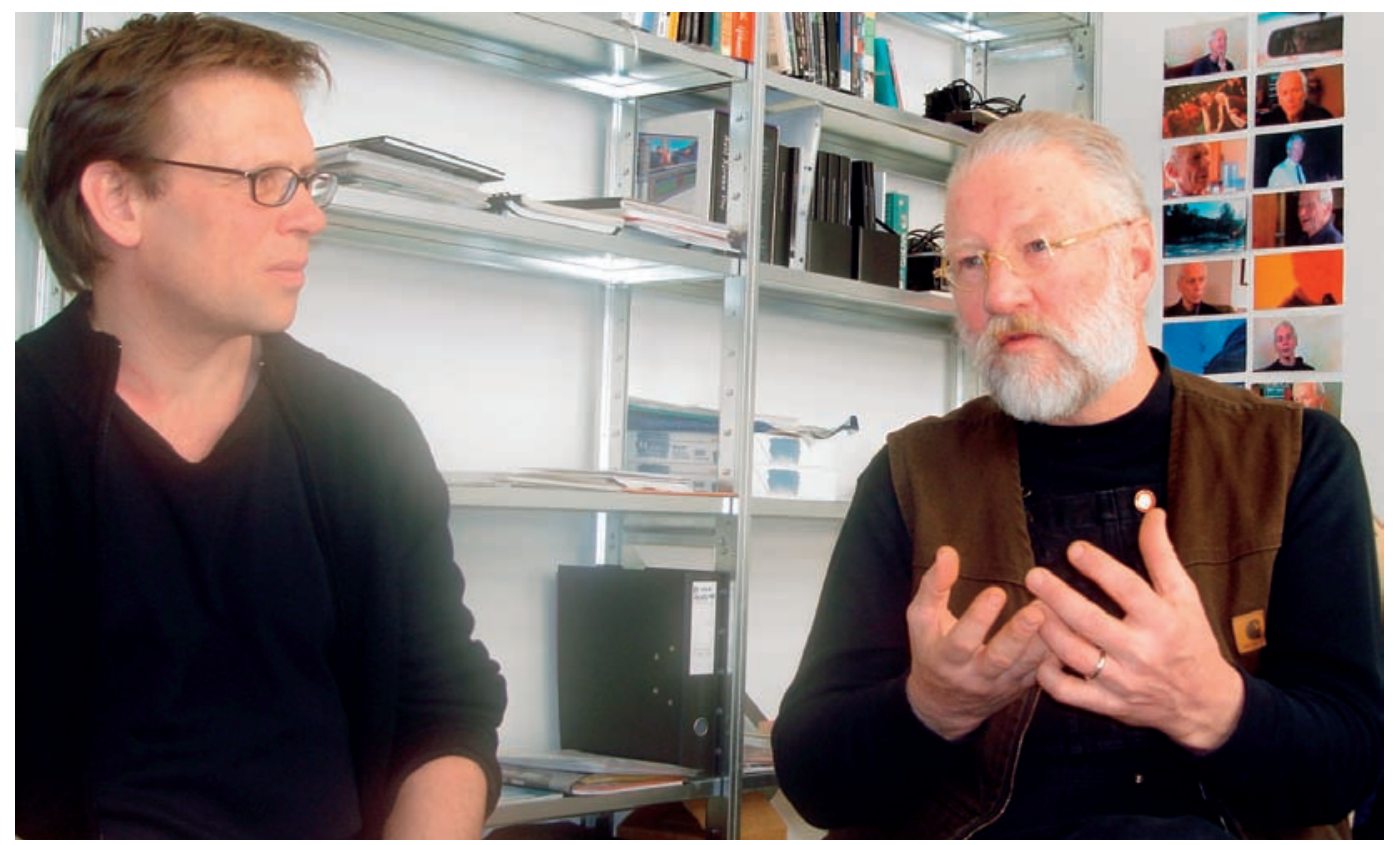

Eric Chapin (rechts): Die Logik der Psychose verstehen. 
Litschig zu Beginn des Films. Es sind ja keine einfachen Persönlichkeiten, die Sie porträtieren.

Hagen: Im Fall von Karen habe ich - haben wir tatsächlich lange überlegt: Kann man das? Darf man das? Von verschiedenen Seiten wurde mir

\section{«Derjenige, der die Diagnose stellt, ist gar nicht so weit von dem entfernt, der sie erhält»}

gesagt, eine Rückkehr an den Ort des traumatischen Geschehens gehe nur in Begleitung eines Psychotherapeuten. Eric [Chapin, die Red.] war es schliesslich, der gesagt hat: «Wenn Karen sich das ohne therapeutische Begleitung zutraut, dann sollte sie dieses Vertrauen auch von eurer Seite erhalten.»

Chapin: «Trust your mind»- das ist in der Tat eine zentrale Botschaft, die wir unseren Patientinnen und Patienten zu vermitteln versuchen. Hagen: Wie Sie gesagt haben, sind die Protagonisten des Films keine «einfachen Persönlichkeiten». Für mich war entscheidend, Leute zu finden, die ein Interesse an meinen Fragestellungen haben, die den Dingen auf den Grund gehen wollen. Die Entstehung des Films war ein langwieriger Prozess, es gab Krisen und Momente, in denen alles ins Stocken geriet. Der Schlüssel zum «Erfolg» war letztlich, dass ich mit jeder der involvierten Personen eine persönliche Beziehung aufgebaut habe. Dadurch entstand die Vertrauensbasis, auf der sich das Projekt realisieren liess. 